\title{
REVIEW ON UNIDIRECTIONAL PLANAR MONOPOLE ANTENNA
}

\author{
Miss. P. D. Swami \\ Department of Digital Communication \\ M.B.E.S. COE, Ambajogai, Maharashtra, India.
}

\begin{abstract}
Antennas are playing a vital role in space of modern wireless communication while delivering a widest range of bandwidths as per the requirement of the application which is cheaper and compacts in size also. Direction of radiation is an important factor in antenna to gain more desirable connectivity with reduced set of energies, so different shapes with different structures and with different meta material are comes in scenario by the researchers. Sometimes the application need to be focus on unidirectional antenna for high energy gain and high performance. This paper reviews and studies different aspects of unidirectional planar monopole antenna and also compares them.
\end{abstract}

Keywords - transmitarray, unit cell element, antenna beamsteering

\section{INTRODUCTION}

In recent the increasing demand of wireless communication forcing the researchers towards optimizing the performance of wireless systems and its setups. This growth in users continuously starves for the bandwidth and connectivity for online services and communications. Usually unidirectional planar monopole antennas have several advantages as ease to fabricate, low cost and also they have narrow bandwidth. In now a days many techniques have been implemented to raise the radiation performance of these antennas according to application needs. The number of researches are intended to manufacture a conventional monopole antennas with various planar configurations in last few decades [1]-[4]. As a result, different parameters of the antenna such as beam pointing, beam shaping or polarization purity can be controlled and the performance of the system can be improved. In monopole antenna structure is placed perpendicular to the conductive surface usually called as ground plane for unidirectional radiation [1], [4]. Three monopoles and their images in a perfect ground plane are shown in figure 1. Becoming a planar they are easily fits with other peripherals supporting higher degree of modularity to the system. Studies with different geometrical structures such as ellipse, arc-shape, rectangle, bow tie, diamond and trapezoid have been observed with various frequency sets. This narrow radio wave beam width can be realized using electrically dense material on one side to

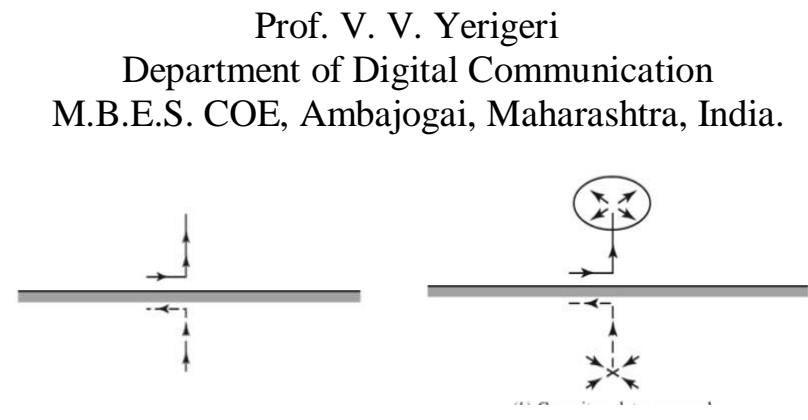

(a) Monopole Antenna

(b) Capacitor plate monopole

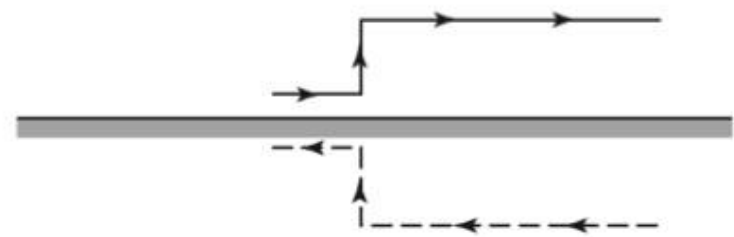

(c) Transmission line monopole

Fig. 1. Monopole antennas over perfect ground plane with their images (dashes)

radiate powerfully on unidirectional whereas less radiation on the other side of the monopole. In [5] unidirectional planar monopole ultra-wideband (UWB) antenna is realized by using a new feed structure which is implemented without modifications in the ground plane or the monopole. Unidirectional radiation of the planar monopole antenna can be achieved by using a wrench-shaped feeding structure with high frequencies. The antenna manufactured on a $20 \times 30 \mathrm{~mm}$ FR4 substrate to cover a frequency bandwidth from 3.1 to over $14 \mathrm{GHz}$ for a voltage standing wave ratio $\leq 2$.

To optimize the radiation patterns and to achieve increased bandwidths, a number of modified shapes of the abovementioned shapes have also been investigated and the field is still being explored to add something new every day. In [6], a wideband unidirectional circularly polarized (CP) antenna of a simple structure that is suitable for array application is achieved by using L-shaped radiator. This L-shaped monopole structure is formed by twofold bends over a large ground plane. This design realizes a circularly polarized unidirectional radiation. In [7] a printed monopole ultra-wideband antenna with reduced ground plane effect is presented. The ground plane dependence of the antenna is decreased by reducing a slot inside the radiator, after which introducing a strip inside the slot, to lessen the current distribution on the ground plane at a required frequency. The antenna is fabricated on a small 
FR4 substrate with an area of $25 \mathrm{~mm} 2$; it achieves an impedance bandwidth of 3.6 to over $11 \mathrm{GHz}$.

Planar monopole antennas yield large impedance bandwidths. The capacity of antenna is mainly depends on the width of the plate, diameter, the feeding probe and also variations of the heights of the monopole. Planar antennas observed to provide multi frequency operations, with height of only about 0.5 times of the lowest operating frequency [8].

Many wireless systems may operate in two or more frequency bands. Planar monopoles bandwidth can be increased in so many ways. By reducing the square edge near ground plane we can acquire a momentous growth in impedance bandwidth and to optimize bandwidth feed gap must be improved in monopole which in fact affects the impedance characteristics [9].

By applying the concept of soft and hard surfaces, a vertical monopole is made to be radiate unidirectional by erecting over a large ground plane in [10-12].

The remaining paper is organized as follows. Construction of planar monopole antenna, its working principle, its features according to shape and size are reviewed in this paper. Various designs and shapes are described in section II. Conclusions are given in section III.

\section{Design And Analysis of Planar Monopole ANTENNA}

Parametric study of the planar monopole antenna is necessary to understand the performances under various aspects of designs and their respected observations. Due to the various features like light weight, low volume, thin size the microstrip monopole antenna has become the first choice of the manufacturer possessing very large bandwidth capability whereas a simple microstrip antenna suffers from narrow impedance bandwidth and low power-handling capability like limitations.

\section{A. Rectangular and square Monopole Design}

Typically these antennas are exhibiting monopolar radiation and observe small change with frequency over the impedance bandwidth. In [13] authors have analysed the formula for broadband square planar monopole to calculate the lower edge frequency of the impedance bandwidth using an equivalent cylindrical monopole and also to calculate upper edge frequency they have used a method-of-moments analysis which employs wire gridding.

A coplanar waveguide (CPW) fed rectangular-shaped monopole antenna is presented in [14] having symmetrical meandered slots on ground plane and operates at $2.4-2.5 \mathrm{GHz}$ for WLAN/Bluetooth band and 3.5-10.9 GHz for UWB band. The experiment achieved omnidirectional and doughnut-shape radiation pattern in $\mathrm{H}$-plane and $\mathrm{E}$ plane respectively, for entire operating bandwidth.

A novel printed square wideband monopole antenna loaded with periodic parallel-plate lines having observed -
$10 \mathrm{~dB}$ performance impedance bandwidth as $1.90 \mathrm{GHz}$ from 2.16 to $4.06 \mathrm{GHz}(61.1 \%)$, covering WLAN $(2.4-2.484 \mathrm{GHz})$, mobile WiMAX (2.5-2.69 GHz), WiMAX (3.4-3.69 GHz), and -band $(2-4 \mathrm{GHz})$ satellite communication service is experimented in [15] by combining a monopole and the periodical structure.

These are omnidirectional antennas in which impedance bandwidth is depends upon various parameters like height, width and distance from the ground plane. To increase the performance of these design structures one can use tridentshaped feeding strip technique to feed the square monopole giving $10 \mathrm{~dB}$ bandwidth of $10 \mathrm{GHz}$ with the lower frequency of $1.376 \mathrm{GHz}$.

More recently, it has been shown that, although the square monopole provides smaller bandwidth than the circular monopole, its radiation pattern suffers less degradation within the impedance bandwidth.[28]

\section{B. Circular monopole Design}

The second design technique to be studied is circular monopole design known by its gradual bevelling near the feeding area. These type of antennas mostly observed to be giving a very broad bandwidth [16] as compared to all the other monopole antenna designs. Change in the input impedance from one mode to another mode is very small due to the bandwidth associated with various mode is very large.

A loop ultra wideband monopole antenna can be obtained by removing the metal from the interior radiator of an ultrawideband circular monopole antenna with a single feeding strip which keeps the radiation characteristics of the antenna as it is. At higher frequencies an enhanced omnidirectional behaviour observed in the circular loop monopole antenna [17].

$\mathrm{Wu}$, Jin and Geng in [18] proposed two quasi-circular planar monopole antennas with rectangular and trapezoidal grounds reporting return-loss bandwidths of better than $10 \mathrm{~dB}$ from 1.3 to $18.4 \mathrm{GHz}$ and 1.1 to $13.5 \mathrm{GHz}$, respectively. Simulated and experimental results proved that the antenna with trapezoidal ground has improved radiation compared to that with the rectangular ground.

In 2001, a wideband orthogonal square monopole antenna with semi-circular base is designed in [19] which operates at 1.4 to $7.5 \mathrm{GHz}(137 \%)$ for VSWR less than 2. The elliptical monopole fed along the major axis were reported to yield maximum bandwidth about 10:1. [28]

\section{Equilateral Triangular Monopole Design}

In [20] a new compact microstrip-fed printed equilateral triangular monopole with dual-frequency operation is described. Various frequency ratios, within the range 1.371.75 , of the two operating frequencies can be obtained by varying the trapezoidal slit dimensions of the triangular patch. Omnidirectional radiation patterns of the two operating frequencies are given for the proposed antenna. 


\section{International Journal of Engineering Applied Sciences and Technology, 2020 \\ Vol. 5, Issue 1, ISSN No. 2455-2143, Pages 531-535 \\ Published Online May 2020 in IJEAST (http://www.ijeast.com)}

The maximum thickness of the antenna is decreased to $1.524 \mathrm{~mm}$ by using the proposed design method [21]. The experiments demonstrates that the antenna delivers worthy Omni directivity radiation pattern, wide bandwidth about $112 \%$ covers $3.2 \mathrm{GHz}-11.35 \mathrm{GHz}$, low crosspolarization, consistent gain, and high-radiation efficiency (over $93 \%$ ) within the entire working frequency band.

Tab monopole antenna is developed which is analogous to a narrow band triangular monopole antenna in [22]. The bandwidth is amplified from $\approx 550 \mathrm{MHz}(1.75 \mathrm{GHz}$ to $2.3 \mathrm{GHz})$ up to $6 \mathrm{GHz}(1.9 \mathrm{GHz}$ to $7.9 \mathrm{GHz})$, for return loss better than $10 \mathrm{~dB}$, by defecting the ground plane. Impedance bandwidth of $6.8 \mathrm{GHz}$ showing bandwidth ratio of $>4: 1$ is measured for return loss better than $7 \mathrm{~dB}$.

\section{Hexagonal Monopole Design}

The experiment with two different feed arrangements (vertices and side feed) is observed with various parameters to examine the effect of feed gap on bandwidth. The simulation results are analysed to study the variations in bandwidth and radiation patterns. Coplanar waveguide-fed printed hexagonal monopole is configured in [23] giving ultra-wide impedance bandwidth ratio of 15.3:1 for a voltage standing wave ratio of $2: 1$.

Some UWB applications requires hexagonal monopole antenna but present solutions have coplanar waveguide fed structures. Symmetrical hexagonal shaped monopole antenna with reduced size with defected ground plane fabricated in [24] and also impedance, radiation and gain characteristics of the proposed structure are studied. Symmetrical hexagonal shaped radiating patch is developed in their novel small microstrip antenna using FR4 substrate. This makes antenna cheaper and easy to manufacture. The antenna also consists of three rectangular slots embedded in a ground plane operating at band of $3.1-12.18 \mathrm{GHz}$ with a return loss of $-10 \mathrm{~dB}$ or more with impedance bandwidth $(\mathrm{S} 11<-10 \mathrm{~dB})$ of $9.03 \mathrm{GHz}$ and a maximum gain of $5.1 \mathrm{dBi}$.

In [25] researchers presented a compact and low profile microstrip fed wearable monopole antenna which operates in 3.7 $\mathrm{GHz}$ to $10.7 \mathrm{GHz}$. The antenna makes use of hexagonal patch with slot as radiating element and blue jeans as substrate.

\section{E. OtherMonopole Designs}

The paper [26] proposes a T-shaped aperture-coupled microstrip-fed, triangular patch antenna which is physically smaller than the aperture coupled rectangular patch antenna or microstrip slot antenna and achieved a $44.5 \%$ bandwidth for VSWR $\leq 2$.

In 2006 Ge, Esselle and Bird depicts a compact E-shaped patch antenna along with corrugated wings. A $25 \%$ reduction in antenna size was achieved by introducing corrugations into the two side wings of the E-shaped patch worked satisfactorily from 5 to $6 \mathrm{GHz}$ [27]. It is found that a $\mathrm{T}$ shaped patch antenna geometry provides wider bandwidth as compared to the Rectangular patch antenna. Guo, Luk and Lee in 2002 demonstrated a broadband and dual-frequency shorted Ushaped microstrip antenna. The U-shaped patch has two different lengths to produce staggered resonant frequencies. The lower band frequency was controlled by the width of Ushaped slots, while the higher band frequency was controlled 10 by the length of a U-shaped slot. Wide impedance bandwidth $(20 \%)$ was obtained for a given substrate material and thickness.

A wideband planar monopole antennas with unidirectional radiation properties, reduced size, easy manufacture and cost effective features are presented in [29]-[34] for compact wireless devices for various applications like microwave-based medical diagnostic systems, X-band application, Heart Failure Detection System, etc.

Removing of metal from the interior structure of the square, circular and elliptical monopoles does not affects their impedance bandwidth performance for square and circular monopole and increased the bandwidth of the elliptical one. The circular and elliptical monopole antennas with metal removed showed better omnidirectional behavior at higher frequencies. Some of the studied monopole antennas were built and tested in [17].

\section{CONCLUSION}

In this paper we have studied antennas with different parameters like size, shapes and structures, etc. according to manufacturing a planar monopole antenna and for different industrial applications. Different design techniques with their benefits over other designs with impedance bandwidth and radiations patterns are compared and described. Paper is more focused on rectangular, square, circular, triangular, hexagonal and few monopole designs. It is found that as compared to all designs a circular shape design gives a better performance gain according to the most of the reviewed papers. So as discussed in above paper we have concluded that a best results can be observe with circular shape antenna but sometimes it is necessary to follow a specific design structure according to the required application field of antenna, for example sometimes we required an unidirection or omnidirection radiation pattern, or sometimes required for longer or shorter distances, or sometimes required cost efficiency.

\section{REFERENCE}

[1] Kishore, Kamal (2009). Antenna and Wave Propagation. IK International Ltd. p. 93. ISBN 9380026064.

[2] Weiner, Melvin M. Weiner (2003). Monopole antennas. USA: CRC Press. pp. vi. ISBN 0-8247-4844-1.

[3] J. R. Panda, A. S. R. Saladi, Rakhesh Singh Kshetrimayum, (2011) "A Compact Printed Monopole 


\section{International Journal of Engineering Applied Sciences and Technology, 2020 \\ Vol. 5, Issue 1, ISSN No. 2455-2143, Pages 531-535 \\ Published Online May 2020 in IJEAST (http://www.ijeast.com)}

Antenna for Dualband RFID and WLAN Applications, " Radioengineering, vol. 20, no. 2, June 2011, pp. 464-467.

[4] P. Gundeti, T. K. George, "Performance analysis of a compact directional monopole antenna", IEEE Applied Electromagnetic Conf. pp. 1-4.

[5] MS Ellis, Z Zhao, J Wu, Z Nie, QH Liu, "Unidirectional planar monopole ultra-wideband antenna using wrenchshaped feeding structure", Electronics Letters 50 (9), 654655 .

[6] D. J. Bisharat, S. Liao, Q. Xue, "Wideband unidirectional circularly polarized antenna with L-shaped radiator structure", IEEE Antennas and Wireless Propag. Lett., vol. 16, pp. 12-15.

[7] Mubarak Sani Ellis, Zhiqin Zhao, Jiangniu Wu, Zaiping Nie, Qing Huo Liu, (2017) "Small planar monopole ultrawideband antenna with reduced ground plane effect", IET Microwaves, Antennas \& Propagation 9 (10), 1028-1034.

[8] Shun-Yun Lin (2003) "Multi-band Planar Monopole Antenna for Handset" 0-7803-7846, IEEE.

[9] Naffall Herscovici and Christos Christodouloc, (2003) "Wideband monopole antennas for multi-band wireless systems" IEEE Antennas and Propagation Magazine , Vol. 45, No.2, April 2003.

[10]D. J. Bisharat, S. Liao, Q. Xue, (2017) "Wideband unidirectional circularly polarized antenna with L-shaped radiator structure", IEEE Antennas and Wireless Propag. Lett., vol. 16, pp. 12-15.

[11]J.-G. Lee, J.-H. Lee, (2018) "Directional monopole antenna using half PMC and PEC ground plane", Microw. Opt. Technol. Lett., vol. 60, pp. 979-983.

[12] Kildal, P.-S, (1990) "Definition of artificially soft and hard surfaces for electromagnetic", IEEE Trans. Antennas Propag., vol. 38, no. 10, pp. 1537-1544.

[13]Ammann, M.J. (2000)., "Impedance bandwidth of the square planar monopole", Microwave and Optical Technology Letters. 24. 185 - 187.

[14] S. Bhaskar, R. S. Brar and A. K. Singh, "Compact planar rectangular monopole antenna for Bluetooth and UWB Applications," 2016 IEEE Uttar Pradesh Section International Conference on Electrical, Computer and Electronics Engineering (UPCON), Varanasi, 2016, pp. 138-141.

[15] W. Wang, M. Su, J. Gao, and Y. Liu, (2017), "Bandwidth Extension of a Printed Square Monopole Antenna Loaded with Periodic Parallel-Plate Lines", vol. 2017, International Journal of Antennas and Propagation, 16875869.

[16]Honda, S., Ito M., Seki H., and Jingo Y., "A Disc Monopole Antenna with 1:8 Impedance Bandwidth and Omnidirectional Radiation Pattern," Proc. ISAP, Sapporo, Japan, 1992, pp. 1145-1148.
[17]A. Mohamed, L. Shafai, (2011), "Performance Study on Modern Ultra Wideband Monopole Antennas", Ultra Wideband Communications: Novel Trends - Antennas and Propagation, IntechOpen, Available from: https://www.intechopen.com/books/ultra-widebandcommunications-novel-trends-antennas-andpropagation/performance-study-on-modern-ultrawideband-monopole-antennas.

[18] Wu, Qi \& Jin, Ronghong \& Geng, Junping. (2009). Ultrawideband quasi-circular monopole antennas with rectangular and trapezoidal grounds. Microwaves, Antennas \& Propagation, IET. 3. 55 - 61.

[19]P. V. Anob, K. P. Ray and G. Kumar, "Wideband orthogonal square monopole antennas with semi-circular base," IEEE Antennas and Propagation Society International Symposium. 2001 Digest. Held in conjunction with: USNC/URSI National Radio Science Meeting (Cat. No.01CH37229), Boston, MA, USA, 2001, pp. 294-297 vol.3.

[20]H. Chen, (2002), "Microstrip-fed dual-frequency printed triangular monopole," in Electronics Letters, vol. 38, no. 13, pp. 619-620.

[21]Song, Zhiwei \& Zheng, Hongxing \& Wang, Mengjun \& Li, Yan \& Song, Tao \& Li, Erping \& Li, Yongjian. (2019). Equilateral Triangular Dielectric Resonator and Metal Patch Hybrid Antenna for UWB Application. IEEE Access. PP. 1-1. 10.1109.

[22] Singh, Sunil \& Sharma, Mahaveer \& Kashyap, Nitesh. (2016). A modified planar triangular monopole antenna for wide band applications. 598-602.

[23] Ray, Dr \& Tiwari, S.. (2010). Ultra wideband printed hexagonal monopole antennas. Microwaves, Antennas \& Propagation, IET. 4. 437 - 445.

[24] Roy, Bappadittya \& Chowdhury, Santosh \& Bhattacharjee, A.K.. (2019). Symmetrical Hexagonal Monopole Antenna with Bandwidth Enhancement Under UWB Operations. Wireless Personal Communications.

[25] G. Krishnaveni, B. Manimegalai and B. Saravanya, "A hexagonal monopole textile antenna for UWB applications," 2015 International Conference on Innovations in Information, Embedded and Communication Systems (ICIIECS), Coimbatore, 2015, pp. 1-6.

[26] Y. Jang, (2001), "Wide-Band T-shaped microstrip-fed twin-slot array antenna", ETRI Journal, vol. 23, no. 1, pp. 33-38.

[27] Y. Ge, K. P. Esselle and T. S. Bird, (2006) "A compact Eshaped patch antenna with corrugated wings," in IEEE Transactions on Antennas and Propagation, vol. 54, no. 8, pp. 2411-2413.

[28] Hammoud, Poey P., and Colomel F., (1993) "Matching the Input Impedance of a Broadband Disc Monopole," Electronics Letters, Vol. 29, pp. 406-407. 
[29] M. S. Ellis, A. Ahmed, J. J. Kponyo, J. Nourinia, C. Ghobadi and B. Mohammadi, (2019) "Unidirectional Planar Monopole Antenna Using a Quasi-Radiator," in IEEE Antennas and Wireless Propagation Letters, vol. 18, no. 1 , pp. 157-161.

[30]A. T. Mobashsher and A. Abbosh, (2014) "Slot-Loaded Folded Dipole Antenna With Wideband and Unidirectional Performance for L-Band Applications," in IEEE Antennas and Wireless Propagation Letters, vol. 13, pp. 798-801.

[31]C. Locker, T. Vaupel and T. F. Eibert, (2005) "Radiation efficient unidirectional low-profile slot antenna elements for X-band application," in IEEE Transactions on Antennas and Propagation, vol. 53, no. 8, pp. 2765-2768.

[32]C. Tang and Q. Xue, (2013) "Vertical Planar Printed Unidirectional Antenna," in IEEE Antennas and Wireless Propagation Letters, vol. 12, pp. 368-371.

[33] S. A. Rezaeieh and A. M. Abbosh, (2014) "Wideband and Unidirectional Folded Antenna for Heart Failure Detection System," in IEEE Antennas and Wireless Propagation Letters, vol. 13, pp. 844-847.

[34]C. Wang and T. Sun, (2011) "Design of a Microstrip Monopole Slot Antenna With Unidirectional Radiation Characteristics," in IEEE Transactions on Antennas and Propagation, vol. 59, no. 4, pp. 1389-1393. 